\title{
Optimal Design of Bracket Isolator Stiffness in Vibration and Shock Environment
}

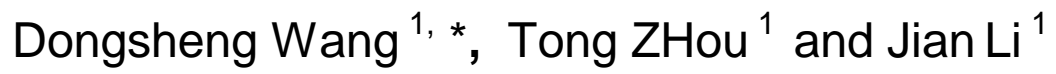 \\ ${ }^{1}$ Institute of Systems Engineering, CAEP, Mianyang Sichuan, China
}

\begin{abstract}
Keywords: vibration; shock; isolator; stiffness; optimal design
Abstract. In this paper, the bracket of certain electric equipment was designed by considering the vibration and shock environment. According to the design requirement of vibration and shock, the stiffness of the isolator was set as the design variable. The mathematic optimal model of getting the largest stiffness of isolator was built to make the relative displacement smaller. The optimal question was solved by using the ANSYS. Both the mode superposition method and the Newmark integrating method were used to get the response of stochastic vibration and chock. The optimal result provided gist for the isolator design of the electric system.
\end{abstract}

\section{Introduction}

The electronic equipment is an important part of planes, delivery vehicles, satellites and other aviation and aerospace aircrafts. However, in dealing with electric devices in the rockets which are often affected by vibration impacts induced by the rocket firing, stage separation and aerodynamic noise, etc. If the adherent resistance against vibration and impact is not strong enough, the electronic device would be occasionally damaged or even failed in worse cases. As one of lessons learnt from the existing experiences in design of aviation and aerospace aircrafts, due to the constraints of the mass, quality, structural performance and manufacturing process, the desirable and satisfactory result could not be provided if only by reinforcing the resistance against environmental vibration and impact[1]. Thus, one of the common criteria for structural design of the electronic device is to minimize the response level under a certain loading condition. As a frequent solution for this purpose, there's often an additional shock absorber or damper in the supporting frame of the structure[2 5]. Due to the inevitable effect induced by vibration or/and impact, the adaptability for both of those factors should have to be taken into account at the same time.

As a popular option in practice, the isolating technologies have been reported in many fields. There have been many examples, some of which are to be given in following. The study on shock resistance performance of a shock reduction system for vessels has been reported by Weng Xuetao, et., al[6]; the result of the study on the impact characteristics of damping element has been reported by Wen Jianming, et.al[7]; one of the designs of shock isolators for rapier looms has been proposed by Mao Nanmin[8]; a simulated study on isolation system for nonlinear vibration and shock has been made by Liu Yong, et.al[9]; the damping effects and data range of the vibration and shock isolation has been analyzed by Zhan Jiakai[10]; the results of the study on shock and vibration has been presented by Chen Jihong[11]. According to the existing results, the reasonable absorber design could contribute to reduce response level to the vibration and shock significantly.

As indicated by statistical results of failures in the vibration and shock environmental tests for a certain electronic system, most of failures are caused by structural strength and functional failure. In light of the understanding that most of failures are caused by the over-big response, the authors began their optimal stiffness design efforts for the bracket (supporting frame) under vibration and shock environment. This design took into account both mechanical conditions including that of the wide-band random vibration and that of half-sine shock. In dealing with these, an optimal mathematical model was established, where, the damper's stiffness was set to be the design variable, the constraints were set in accordance with root mean square and the maximal value of the shock response of random vibration of the important part of supporting frame, and the reciprocal of the 
damper's stiffness was set to be the objective function. Based on this model, optimization design based on the ANSYS parameter language was made to find out the solution.

\section{Theoretical Methods}

In this paper, the platform for the optimized design was ANSYS, and the random vibration was analyzed with mode superstition method, which superposed all the structural responses to provide the structural response, based on the statistical calculation of the structural responses for each order of the modes. The shock analysis was made with Newmark time integration method. In linear structural response analysis, at a randomly given time $(\mathrm{t})$, the dynamic equation was presumed to be sieries of static equilibrium equations that took account effects of inertial force and damping force. Then, in accordance with ANSYS program, Newmark time integration method was used to solute these equations at a discrete time spot. In this way, the shock response of the structure could be obtained. Besides, in this paper, the complete method (Full) was used to calculate the shock response, which requires a complete system matrix computation. And the optimization method used in this paper was the 1st order optimization method. Such a method is based on the sensitivity of the object function to the design variables, which is a favorite option for hi-precision optimization analysis but poor in computing efficiency. With an additional penalty function, the object function would be converted the problem into an unconstraint and most optimized problem, which could then be settled with the sequential unconstraint minimization technique (SUMT).

\section{Development of Optimization Model}

As shown in Fig.1, the finite element model (FEM) of the bracket consisted of isolator, bracket and electronics, with a total mass of $5.57 \mathrm{~kg}$. In the mathematical model, the stiffness of isolator $(\mathrm{k})$ was set to be the design variable. In the model, some constraints were deliberately set up, including the roots of mean square of the random vibrating acceleration response[Arms(110Z) and Arms(203Z)] and the maximum acceleration response [Amax(110Z) and Amax(203Z)] induced by semi-sine shock along Z-direction of No. 110 node and No.203 node in the bracket. Besides, the reciprocal of the stiffness $(1 / \mathrm{k})$ was set to be the object function. All of the additional constraints were made to provide a bracket system not only to fulfill the constraint requirements but also to mitigate the relative displacement of the system in the accelerating field. As for the optional damping ratio for the isolator in engineering, the common range is proposed to be within $0.08 \sim 0.2$, which was set to be 0.15 in this paper.

For a bracket system, the isolator stiffness optimization under random vibration and shock load could be described as follows.

Firstly, find out the solution of the design variable, i.e. $\mathrm{P}=\mathrm{k}$. Then, take account the random vibration and semi-sine shock loading, and make the following constraint conditions available:

$$
\begin{aligned}
& 5.0 \times 10^{4} \mathrm{~N} / \mathrm{m} \leq k \leq 1.2 \times 10^{5} \mathrm{~N} / \mathrm{m} ; \\
& A_{\text {rms }}(110 \mathrm{Z}) \leq 8 g ; A_{\text {rms }}(203 \mathrm{Z}) \leq 8 g \\
& A_{\max }(110 \mathrm{Z}) \leq 35 g ; A_{\max }(203 \mathrm{Z}) \leq 35 g
\end{aligned}
$$

And finally, we could have an object function as $A(P)=1 / k \rightarrow$ min $。$

\section{Optimized Results}

Based on the understanding that the effects of vibration and shock loading on the installation position of the isolator are in form of base excitation, the installation position was fixed supported (clamped). The random vibration and shock loading were perpendicular to the bracket plate (Z-direction). The RMS of the accelerating power spectrum was $13.85 \mathrm{~g}$, while the shock loading was a semi-sine wave with $8 \mathrm{~ms}$ in pulse width and $45 \mathrm{~g}$ in amplitude. 
The minimum feasible solution of the object function occurred in the ninth optimal step of the optimal solution. As indicated by the parameter comparison before and after optimization in Tab.1, the isolator stiffness after optimization approaches to be reasonable, where all of the constraints are fulfilled and the minimum value of the object function is obtained. The optimal procedure of the object function is given in Fig.2, while the optimal procedures of random vibration acceleration RMS and shock-induced response peak value for No.110 node and No.203 node are given respectively from Fig.3 to Fig.6

\section{Conclusions}

An optimal stiffness design of an isolator in the vibration and shock environment could be used not only to minimize effectively the vibration and shock-induced response, but also to improve the design efficiency.

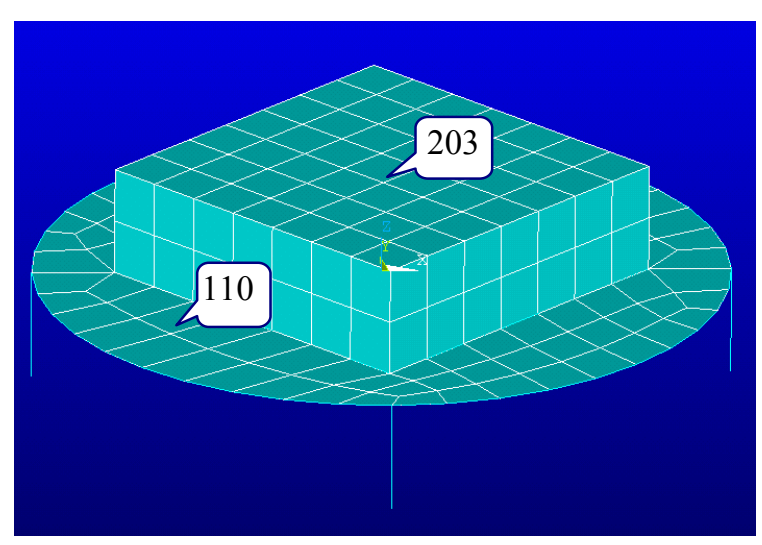

Fig.1 FEM of bracket

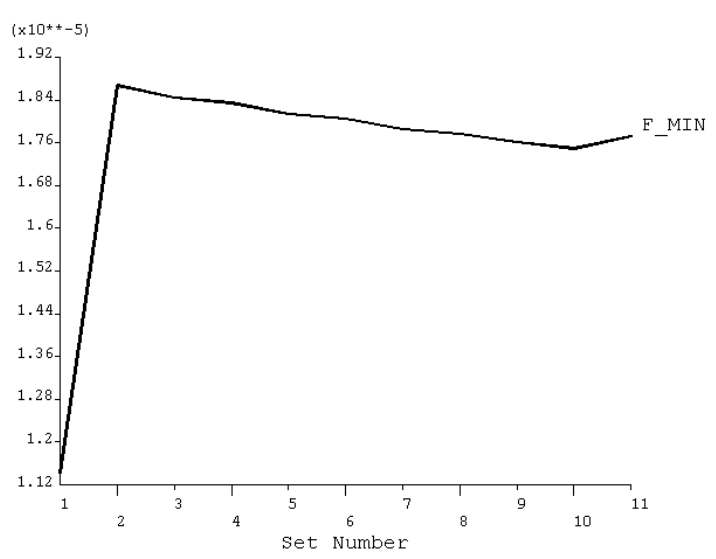

Fig. 2 Optimal procedure of the object function

Tab. 1 The design variables with and without optimization

\begin{tabular}{c|c|c|c}
\hline Item & unit & Initial value & optimized value \\
\hline$k$ & $\mathrm{~N} / \mathrm{m}$ & 87500 & 56769 \\
\hline$A_{\mathrm{rms}}(110 \mathrm{Z})$ & $g$ & 9.59 & 7.92 \\
\hline$A_{\mathrm{rms}}(203 \mathrm{Z})$ & $g$ & 9.55 & 7.90 \\
\hline$A_{\max }(110 \mathrm{Z})$ & $g$ & 39.85 & 33.30 \\
\hline$A_{\max }(203 Z)$ & $g$ & 42.27 & 35.15 \\
\hline
\end{tabular}

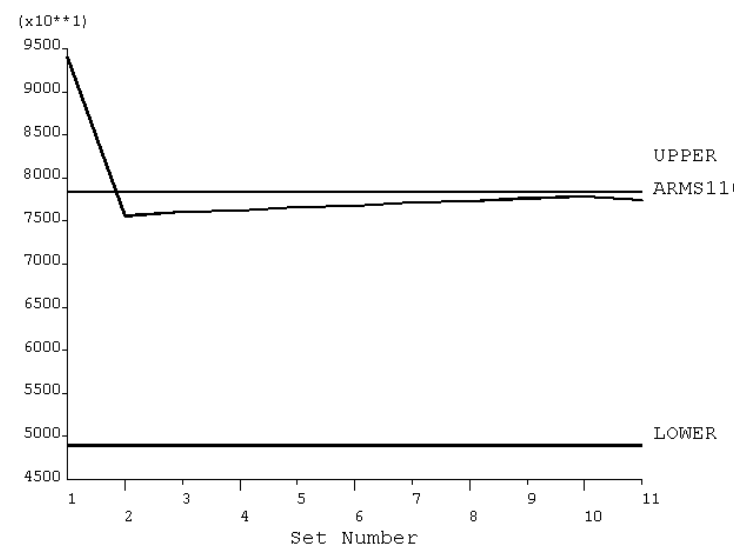

Fig. 3 Optimal procedure of the acceleration of the No.110 node

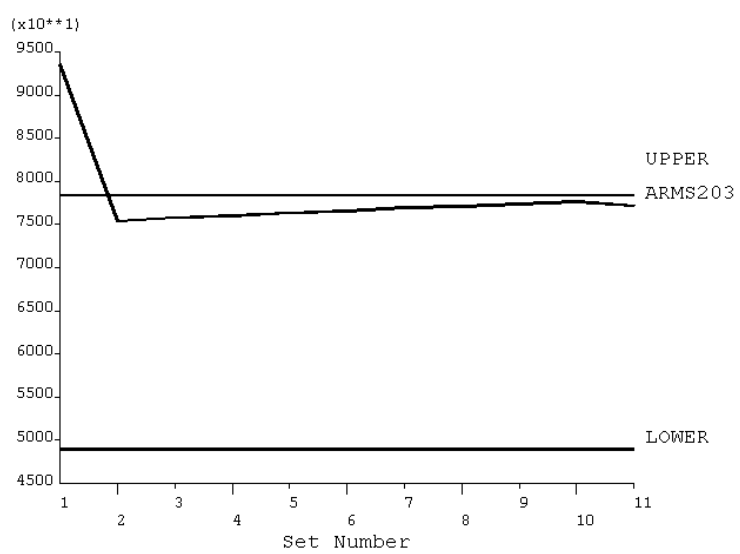

Fig. 4 Optimal procedure of the acceleration of the No.203 node 


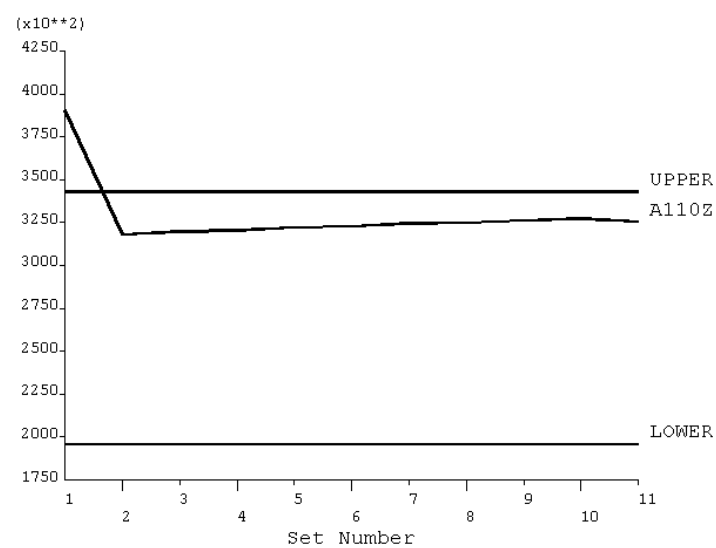

Fig. 5 Optimal procedure of the shock response of the No.110 node

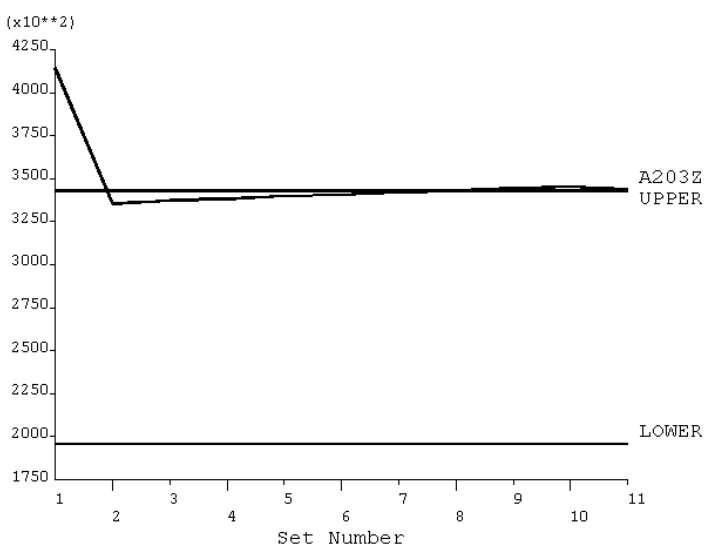

Fig. 6 Optimal procedure of the shock response of the No.203 node

\section{References}

[1] Yanshen Xu,et al. Dynamic Design of Mechanical Systems[M in Chinese].Beijing :Mechanical Industry Press, 1992. In Chinese.

[2] Qingshan Xue. The advance and trends of vibration isolated technique[J in Chinese].Journal of Mechanical Strength, 1994,16(1):37 42. In Chinese.

[3] Chong Chen, Ziheng Wu. Characteristics and application of nonlinear wire-rope vibration isolator[J]. Journal of Sichuan University of Science and Technology,1999,18(1):1 7. In Chinese. [4] Jianyou Sheng. Guarding against vibration and resisting lash design of airborne electronic equipment[J in Chinese].Electronics Machinery Engineering, 1999,2:44 47. In Chinese.

[5] Daoxun Liao, Xiaocheng Huang, Yongzhong Lu. Theory and Method of Dynamic Modeling for Multilayer Vibration Isolation System [ $\mathrm{J}$ in Chinese]. China Mechanical Engineering, 1999, 10(12):1321 1324. In Chinese.

[6] Xuetao Weng,et al. Computation about the Shock Resistance Performance of Vibration Reduction System[J in Chinese]. Noise and Vibration Control,1999,2:16 18. In Chinese.

[7] Jianming Wen, et al. Study on impact characteristic about buffer component[J in Chinese].Noise and Vibration Control,2001,2:18 21. In Chinese.

[8] Nanmin Mao. A new design method of shock isolators[J in Chinese]. Noise and Vibration Control,1997,1:22 25. In Chinese.

[9] Yong Liu, et al. Research on simulation of vibration and shock isolation for nonlinear system[ $\mathrm{J}$ in Chinese].Mechanical Science and Technology, 1999,18(2):204 206. In Chinese.

[10] Jiakai Zhan, et al. Effect and Range of Damping on the Reduction of Vibration and Shock[J in Chinese]. Noise and Vibration Control,1998,3:21 25. In Chinese.

[11] Jihong Chen.The difference of isolation of vibration and shock[ $\mathrm{J}$ in Chinese]. Noise and Vibration Control,1996,1:31 33. In Chinese.]

\section{Dongsheng Wang, tel: 13990134005, E-mail: wangds@caep.cn}

